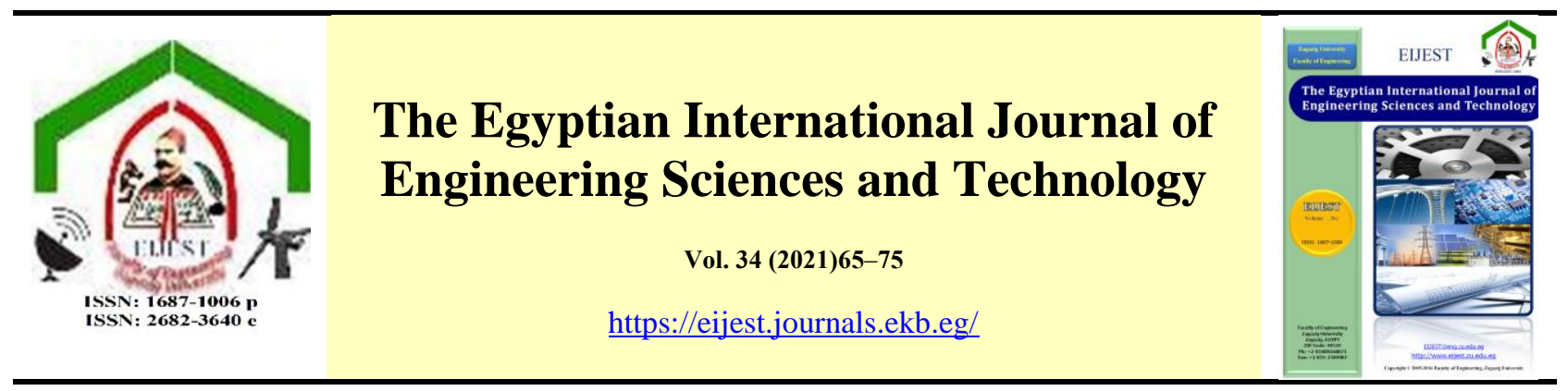

\title{
Designing optimal power system stabilizer for synchronous generator with and without damper windings
}

\author{
Ahmed H. Abd-Elkareem ${ }^{\mathrm{a} *}$, Mohamed A. Elhameed ${ }^{\mathrm{b}}$, Mahmoud M.Elkholy ${ }^{\mathrm{b}}$ \\ ${ }^{a}$ Operation Engineer at new capital power plant, New capital city, Egypt \\ ${ }^{b}$ Associate professor at electrical power and machines Dept, Faculty of Engineering, Zagazig University, Zagazig, Egypt
}

\section{A B S T R A C T}

This paper aims at effective damping of low-frequency oscillations (LFO) associated with synchronous generators (SG).

LFO are excited by system disturbances as sudden change on the load, switching events, and malfunction of the turbine controller.

Poor damping of LFO affects the stability of the generation system, in severe cases may result in instability and damage to prime-movers.

The negative effect of the automatic voltage regulator (AVR) is studied for SG without and with damper windings on the damping of oscillations.

Minimizing the LFO is achieved by incorporating the power system stabilizer (PSS), which adds an additional control signal to AVR. Effective design of PSS to maximize the damping torque while maintaining the stability of SG variables.

The problem is modelled as an optimization problem, the damping ratio of the mechanical modes is the objective function. The single-pole placement technique and the recently developed political optimizer are picked to determine the perfect PI parameters for PSS.

The satisfactory performance of the proposed PSS is appraised by testing it against mechanical power disturbances.

The obtained mathematical analytical expression makes it possible to determine easily the eigenvalues and step response for the speed and electromagnetic torque for SG in MATLAB environment.

\section{INTRODUCTION}

Increasing the electrical load due to overpopulation and manufacturing have created the need to operate the generated systems close to their capacity limits [1].

In addition to the appearance of unforeseen malfunctions of the control devices of the valves that control the power generated and after that the generator loses synchronism with the grid, destroy the prime-mover of the turbine or reduce the lifetime of the generation system.
This causes an unexpected change in power consumption due to switching events.

So, this leads sometimes to weak dynamic response, stability problems, make electromagnetic disturbances and oscillations on the electrical grid [2].

These reasons affect great damage on the generation power systems (SG) and reducing the stability of the electrical network and lead to decrease the life time and breakdown the generation systems [2].

Therefore, this will be considered very expensive economical. Otherwise, some designs for 
synchronous generators have damper windings that help to damp a small fraction of oscillations. Therefore, the use of excitation systems $[3,4]$ like automatic voltage regulator (AVR) is used but it observes a voltage signal from an infinite bus and compared it with a reference signal to adjust and regulate the generated voltage of synchronous generator by controlling the field current to adjust the voltage deviation.

This led to generate additional induced current in field winding counteract the induced current of damper winding, this makes the damping process deteriorate [1].

The PSS [4] is considered that one of the most important systems in SG because it observes the electrical power and frequency of the generator and when any external fault has happened on the grid or sudden change in the loads, it leads to a swing in the power of generator [6].

The PSS generates a stabilizing signal, which produces a torque damping component on the generator rotor and the created torque component must be in phase with speed deviation, produce the required power to support the grid when a sudden change in the loads or external fault in the grid and compensate the oscillation in the system and make it more stable.

Due to the non-linear electrical grid, analysis of the small-signal eigenvalues and dynamic simulation. The Conventional PSS (CPSS) [8] system has been examined and found to be inefficient in conditions of turbulence and large-scale operation, and it cannot dampen low-frequency oscillations.

To repair this system (CPSS), many techniques have been used to contribute to the conclusion of appropriate values for the PI parameters such as particle swarm optimization [9], Fuzzy wavelet neural network [10], Single Neuron based PSS (SNPSS) [11], multi-band PSS using culture-PSO-co evolutionary (CPCE) algorithm [12], participation factor and genetic algorithm [13].

In this regard, this paper addresses the effect of the AVR system on SG is studied and the single-pole placement and the most recent optimization method (political optimizer $[29,30]$ ) are used to achieve the transient stability for SG.

The linearization process is used in park's equation [5] of SG without and with damper winding to express and simulate the problem of small-signal stability [7] at the disturbance load and analysis the dynamic response of damping.
Therefore, in the present paper:

I - modelling of the infinite bus and synchronous generator in(d-q) axis rotor reference and analysis the equations at steady-state and linearization process.

II -Analysis of the effect of AVR system with SG at the oscillations

III - designing the PSS system with the optimal controller to enhancement the dynamic performance of SG.

Iv - compartment the results of eigenvalues and step response for SG without and with damper winding at the effect of excitation systems at load disturbance.

\section{Modeling of synchronous generator and finite bus}

SG has been usually modeled by using the park's equations [1] to express and simulate its physical description and performance to conclude and analyze the relationship between the disturbance on the electromagnetic grid and the dynamic behavior of the generator.so, equations of SG will be analyzed in (dq) axis rotor reference after transformed from (a-b-c) stator reference frame by using park's equations.

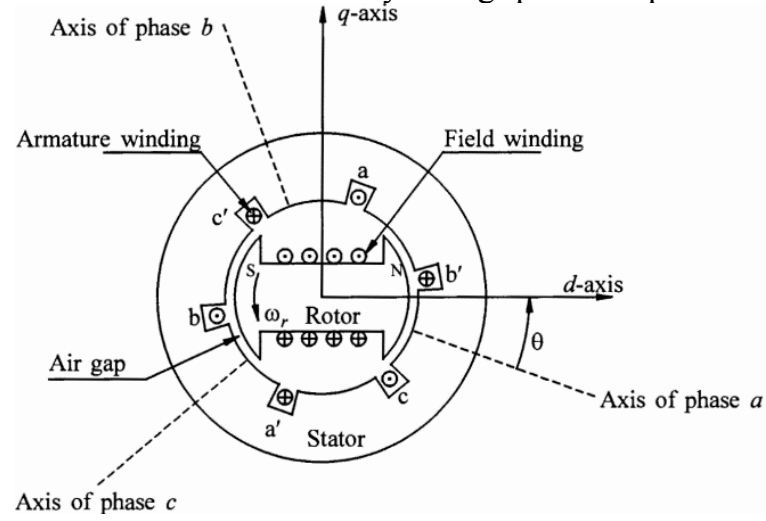

Fig 1. Schematic diagram of SG [2]

the voltage in d-q stator equations $(1,2)$ :

$v_{d s}=-r_{s} i_{d s}+\frac{p}{\omega b} \psi_{d s}-\frac{\omega_{r}}{\omega_{b}} \psi_{q s}$

$v_{q s}=-r_{s} i_{q s}+\frac{p}{\omega b} \psi_{q s}+\frac{\omega_{r}}{\omega_{b}} \psi_{d s}$

the voltage in d-q damper winding equations $(3,4)$ :

$v_{d r}=0=r_{d r} i_{d r}+\frac{p}{\omega b} \psi_{d r}$

$v_{q r}=0=r_{q r} i_{q r}+\frac{p}{\omega b} \psi_{q r}$

The voltage in field winding equation (5)

$v_{f r}=r_{f r} i_{f r}+\frac{p}{\omega b} \psi_{f r}$

The electromagnetic torque and motional equations $(6,7)$ : 
$T_{e}=\frac{3}{2} \cdot \frac{P}{2} \cdot \frac{1}{\omega_{b}}\left(\psi_{d s} i_{q s}-\psi_{q s} i_{d s}\right)$

$T_{l}-T_{e}=\frac{2 j \omega_{b}}{P} p\left(\frac{\omega_{r}}{\omega_{b}}\right)$

Assume SG is connected to the grid through line with resistance $r_{\mathrm{e}}$, inductance $\mathrm{l}_{\mathrm{e}}$.Fig 2

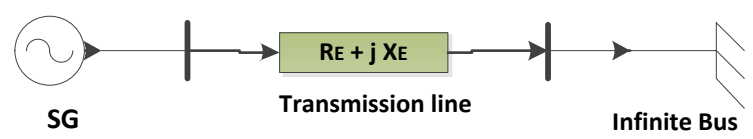

Fig 2. synchronous generator with infinite bus

So, the equations of stator voltage at the grid will be $(8,9)$

$v_{d s}=v_{b} \sin \theta_{b}+\frac{p}{\omega_{b}} x_{e} i_{d s}-\frac{\omega_{r}}{\omega_{b}} x_{e} i_{q s}+r_{e} i_{d s}$ (8)

$v_{q s}=v_{b} \cos \theta_{b}+\frac{p}{\omega_{b}} x_{e} i_{q s}+\frac{\omega_{r}}{\omega_{b}} x_{e} i_{d s}+r_{e} i_{q s}$ (9)

At steady state, the synchronous machine equations terms will be constant so the derivative terms ( $p=$ $\frac{d}{d t}$ ) will be zero, $\omega_{r 0}=\omega_{b}$ synchronous speed and the current of $\mathrm{d}-\mathrm{q}$ damper winding will be zero $i_{d r}=i_{q r}=0$.

the linearization process of SG equations is very important to find the required state, input and output matrixes and analysis the state space for the transfer functions of rotor speed and electromagnetic torque and the dynamic performance of the machine to design the perfect controller so consider a small change from a balanced operating condition of park's equations in physical units.

From the linearization of the stator voltage of q-axis at SG and the linearization process of the stator voltage of q-axis at infinite bus equation. Deducing the equation (10)

$-\left(r_{s}+r_{e}\right) \Delta i_{q s}-\frac{\omega_{r 0}}{\omega_{b}}\left(x_{d s}+x_{e}\right) \Delta i_{d s}$

$+\frac{\omega_{r 0}}{\omega_{b}} x_{m d} \Delta i_{d r}+\frac{\omega_{r 0}}{\omega_{b}} x_{m d} \Delta i_{f r}$

$+\frac{\Delta \omega_{r}}{\omega_{b}}\left(\psi_{d s 0}-x_{e} i_{d s 0}\right)-v_{b 0} \sin \left(\delta_{b 0}\right) \Delta \theta_{r}$

$+\frac{p}{\omega_{b}}\left(-\left(x_{q s}+x_{e}\right) \Delta i_{q s}+x_{m q} \Delta i_{q r}\right)$

$=-v_{b 0} \sin \left(\delta_{b 0}\right) \Delta \delta_{b}+\Delta v_{b} \cos \left(\delta_{b 0}\right)$

And from the linearization of the stator voltage of $\mathrm{d}$ axis at SG equation and the linearization process of the stator voltage of $\mathrm{d}$-axis at infinite bus equation Deducing the equation (11)

$$
\begin{aligned}
& -\left(r_{s}+r_{e}\right) \Delta i_{d s}+\frac{\omega_{r 0}}{\omega_{b}}\left(x_{q s}+x_{e}\right) \Delta i_{q s}+v_{b 0} \cos \left(\delta_{b 0}\right) \Delta \theta_{r} \\
& -\frac{\omega_{r 0}}{\omega_{b}} x_{m q} * \Delta i_{q r}+\frac{\Delta \omega_{r}}{\omega_{b}}\left(-\psi_{q s 0}+x_{e} i_{q s 0}\right) \\
& +\frac{p}{\omega_{b}}\left(-\left(x_{d s}+x_{e}\right) \Delta i_{d s}+x_{m d} \Delta i_{d r}+x_{m d} \Delta i_{f r}\right)
\end{aligned}
$$

$$
=v_{b 0} \cos \left(\delta_{b 0}\right) \Delta \delta_{b}+\Delta v_{b} \sin \left(\delta_{b 0}\right)
$$

Linearization for voltage of $\mathrm{q}$-axis of damper winding equation (12)

$0=r_{q r} \Delta i_{q r}+\frac{p}{\omega_{b}}\left(x_{q r} \Delta i_{q r}-x_{m q} \Delta i_{q s}\right)$

Linearization for voltage of $\mathrm{d}$-axis of damper winding equation (13)

$0=r_{d r} \Delta i_{d r}+\frac{p}{\omega_{b}}\left(x_{d r} \Delta i_{d r}+x_{m d}\left(-\Delta i_{d s}+\Delta i_{f r}\right)\right.$

Linearization for voltage of field winding equation (14):

$\Delta v_{f r}=r_{f r} \Delta i_{f r}+\frac{p}{\omega_{b}}\left(x_{f r} \Delta i_{f r}+x_{m d}\left(\Delta i_{d r}-\right.\right.$

$\left.\Delta i_{d s}\right)$

Linearization of electromagnetic torque and motional equation $(15,16)$

$$
\left.\begin{array}{c}
c_{t}=\frac{3}{2} \cdot \frac{P}{2} \cdot \frac{1}{\omega_{b}} \\
\Delta T_{e}=c_{t}\left(\begin{array}{c}
\left(\psi_{d s 0}+x_{q s} i_{d s 0}\right) \Delta i_{q s} \\
-\left(x_{d s} i_{q s 0}+\psi_{q s 0}\right) \Delta i_{d s}+x_{m d} i_{q s 0} \Delta i_{d r} \\
+x_{m d}
\end{array}\right) \\
\Delta T_{q s 0} \Delta i_{f r}-x_{m q} i_{d s 0} \Delta i_{q r}
\end{array}\right)
$$

Deducing the small change in mechanical torque equation (17) from equations $(15,16)$

$$
\begin{aligned}
& \Delta T_{l} \\
& =c_{t}\left(\begin{array}{c}
\left(\psi_{d s 0}+x_{q s} i_{d s 0}\right) \Delta i_{q s}-\left(x_{d s} i_{q s 0}+\psi_{q s 0}\right) \Delta i_{d s} \\
+x_{m d} i_{q s 0} \Delta i_{d r} \\
+x_{m d} i_{q s 0} * \Delta i_{f r}-x_{m q} i_{d s 0} \Delta i_{q r}
\end{array}\right) \\
& +\frac{2 \cdot j \cdot \omega_{b}}{P} p\left(\frac{\Delta \omega_{r}}{\omega_{b}}\right)
\end{aligned}
$$

Linearization of rotor and speed equation (18)

$$
p \Delta \theta_{r}-\Delta \omega_{r}=0
$$

From equations (10-14), (17-18) respectively are equivalent to the matrix expression to analysis the state space at constant field voltage and dynamic behaviour with damper winding and without damper winding after removing equations $(12,13)$

\section{Modeling of AVR with synchronous generator}

AVR system observes a voltage signal from infinite bus and compare it with a reference signal to adjust and regulate the generated voltage of synchronous generator by controlling the field current. 
this part describes and analysis the linearization of the automatic voltage regulation system and study the effect of AVR on the disturbance on mechanical load. the AVR system (IEEE Type 1(ST1A) excitation system) as shown in Fig.3

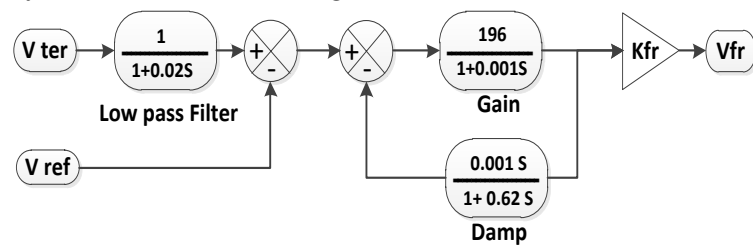

Fig 3. Block Diagram AVR system

Linearization AVR system, all constant values will be removed at small changes from steady state, rewriting the block diagram of AVR after simplifying the system at linearization process. as shown in Fig.4

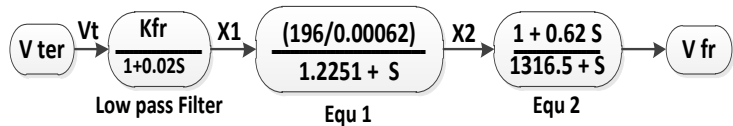

Fig 4. Block diagram after simplifying the exciter system linearization of the generated voltage of synchronous generator $\Delta V_{t}$

$V_{t}=\sqrt{v_{d s}^{2}+v_{q s}^{2}}$

consider a small change from a balanced operating condition on $v_{t}$ :

$v_{t 0}+\Delta V_{t}=$

$=v_{t 0} \sqrt{1+\frac{2 v_{d s 0} \Delta v_{d s}}{v_{t 0}{ }^{2}}+\frac{2 v_{q s 0} \Delta v_{q s}}{v_{t 0}{ }^{2}}}$

(20)

From power series expansion

$\sqrt{1+x}=1+\frac{x}{2 !}+\frac{x^{2}}{4 !}+\cdots$

After eliminate the steady state term and $\Delta V_{t}$ will be divide on $v_{t 0}$ to get the value of small change as per unit, where $v_{t 0}$ is the base voltage

$\Delta v_{t}=\frac{\Delta V_{t}}{v_{t 0}}$

$\Delta v_{t}=a_{-} v_{t} \Delta \delta_{b}+b_{-} v_{t} \Delta v_{b}+c_{-} v_{t} \Delta \theta_{r}+$

$d_{-} v_{t} \Delta \omega_{r}+e_{-} v_{t} \Delta i_{d s}+f_{-} v_{t} \Delta i_{q s}+$

$\Delta i_{d s} \frac{p}{\omega_{b}} x_{e} \frac{v_{d s 0}}{v_{t 0}} \frac{1}{v_{t 0}}+\Delta i_{q s} \frac{p}{\omega_{b}} x_{e} \frac{v_{q s 0}}{v_{t 0}} \frac{1}{v_{t 0}}$

Where,

$a_{-} v_{t}=\left[v_{b 0} \cos \left(\delta_{b 0}\right) \frac{v_{d s 0}}{v_{t 0}}-v_{b 0} \sin \left(\delta_{b 0}\right) \frac{v_{q s 0}}{v_{t 0}}\right] \frac{1}{v_{t 0}}$

$b_{-} v_{t}=\left[\sin \left(\delta_{b 0}\right) \frac{v_{d s 0}}{v_{t 0}}+\cos \left(\delta_{b 0}\right) \frac{v_{q s 0}}{v_{t 0}}\right] \frac{1}{v_{t 0}}$

$c_{-} v_{t}=\left[-v_{b 0} \cos \left(\delta_{b 0}\right) \frac{v_{d s 0}}{v_{t 0}}+v_{b 0} \sin \left(\delta_{b 0}\right) \frac{v_{q s 0}}{v_{t 0}}\right] \frac{1}{v_{t 0}}$

$$
\begin{aligned}
& d_{-} v_{t}=\left[\frac{-x_{e} i_{q s 0}}{\omega_{b}} \frac{v_{d s 0}}{v_{t 0}}+\frac{x_{e} i_{d s 0}}{\omega_{b}} \frac{v_{q s 0}}{v_{t 0}}\right] \frac{1}{v_{t 0}} \\
& e_{-} v_{t}=\left[r_{e} \frac{v_{d s 0}}{v_{t 0}}+x_{e} \frac{\omega_{r 0}}{\omega_{b}} \frac{v_{q s 0}}{v_{t 0}}\right] \frac{1}{v_{t 0}} \\
& f_{-} v_{t}=\left[-x_{e} \frac{\omega_{r 0}}{\omega_{b}} \frac{v_{d s 0}}{v_{t 0}}+r_{e} \frac{v_{q s 0}}{v_{t 0}}\right] \frac{1}{v_{t 0}}
\end{aligned}
$$

From Fig.4, first the transfer function (T.F) for block diagram of $\frac{\Delta x_{1}}{\Delta v_{t}}$ :

$k_{f r} \Delta v_{t}-\frac{p}{\omega_{b}}\left(0.02 \omega_{b} \Delta x_{1}\right)-\Delta x_{1}=0$

substitute $\Delta v_{t}$ from equation (22) into equation (23)

$k_{f r} a_{-} v_{t} \Delta \delta_{b}+k_{f r} b_{-} v_{t} \Delta v_{b}=-k_{f r} e_{-} v_{t} \Delta i_{d s}$

$-k_{f r} c_{-} v_{t} \Delta \theta_{r}-k_{f r} d_{-} v_{t} \Delta \omega_{r}-k_{f r} f_{-} v_{t} \Delta i_{q s}$

$-x_{e} \frac{v_{d s 0}}{v_{t 0}} \frac{1}{v_{t 0}} k_{f r} \frac{p}{\omega_{b}} \Delta i_{d s}$

$-x_{e} \frac{v_{q s 0}}{v_{t 0}} * \frac{1}{v_{t 0}} k_{f r} \frac{p}{\omega_{b}} \Delta i_{q s}$

$+0.02 \omega_{b} \frac{p}{\omega_{b}} \Delta x_{1}+\Delta x_{1}$

second, analysis of the T.F for the block diagram of $\frac{\Delta x_{2}}{\Delta x_{1}}$ :

$\frac{p}{\omega_{b}}\left(\omega_{b} \Delta x_{2}\right)+1.2251 \Delta x_{2}-\left(\frac{196}{0.00062}\right) \Delta x_{1}=0$

Third, analysis of the T.F for the block diagram of

$\frac{\Delta v_{f r}}{\Delta x_{2}}$ :

$\frac{p}{\omega_{b}}\left(\omega_{b} \Delta v_{f r}\right)+1316.517 \Delta v_{f r}-$

$\frac{p}{\omega_{b}}\left(0.62 \omega_{b} \Delta x_{2}\right)-\Delta x_{2}=0$

From equations (10-14), (17,18), (24-26) respectively are equivalent to the matrix expression to analysis the state space at AVR and dynamic behaviour with damper winding and without damper winding after removing equations $(12,13)$

\section{Modeling of AVR and PSS with synchronous generator}

Deduction of the effect of the AVR system from (Fig.11 and Table 4) for SG without damper windings and (Fig.13 and Table 5) for SG with damper windings, AVR system weaken the damping process for SG with damper winding and make unstable dynamic behavior for SG without damper winding at sudden change in the electrical load. Therefore, there must be a system for power system 
stabilizer (PSS) to enhance the dynamic performance and transient stability for SG.

it is considered that effective system in synchronous generator because it observes the electromagnetic power and frequency of the generator and when any external fault is happened on the grid or sudden change in the loads, it leads to swing the power of generator so the system (PSS) generates a stabilizing signal, which produces a torque damping component on the generator rotor and the created torque component must be in phase with speed deviation or produce the required power to support the grid when sudden change in the loads or external fault in the grid .

so, there is a lot of studies and researches about study and analysis the perfect components for PSS system and deducing the best values of PI controller.

in this part of paper discuss and analysis the effect of power system stabilizer (IEEE type PSS1A) on the dynamic performance of synchronous generator with and without damper winding.

PSS use the signal from electromagnetic power of synchronous generator as input as shown in Fig.5

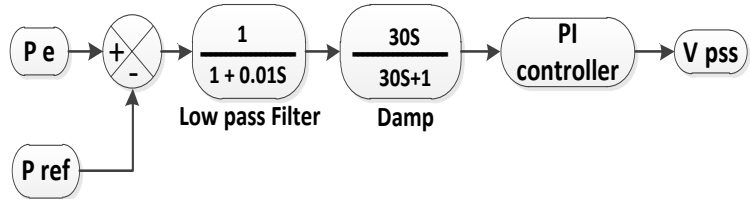

Fig 5. Block Diagram of PSS

PSS is a feedback controller which provides an additional signal to input of summing point with automatic voltage regulation (AVR) as shown in Fig.6

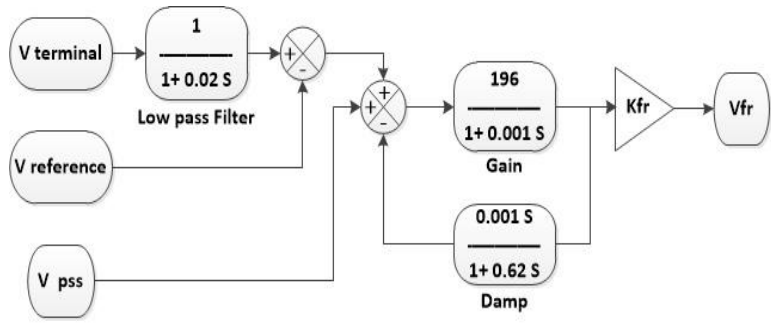

Fig 6. Block diagram of exciter system with PSS signal

In this paper, there are two methods to design PI controller for PSS by using Single Pole Placement and political optimizer method to get the perfect values for $K_{p}, K_{i}$ to achieve the desirable dynamic performance for PSS and compensate the oscillation of system to make the behaviour of SG is more stable at the disturbance in the grid so:

The following steps describe the Single Pole Placement [28]:
1- get the T.F of $\frac{\Delta v_{p s s}}{\Delta p_{e}}$ from the block diagram of PSS that shown in Fig (5).

2- get the T.F of $\frac{\Delta p_{e}}{\Delta v_{p s s}}$ from the state space of synchronous generator with the signal of PSS and $\Delta v_{p s s}$ is selected as input, $\Delta p_{e}$ is selected as output.

3- Equating the sides of the equation from above step1,2, Subsequently, Conclude an equation with one unknown variable $(\mathrm{S})$.

4- assuming the best value of (S) that achieve the desirable dynamic performance of the rotor speed and electromagnetic torque and make sure the value of (S) is the same the eigen value of $\left(\Delta \omega_{r}, \Delta \theta_{r}\right)$.

5- deduce the pi controller parameter $\left(K_{p}, K_{i}\right)$.

In the second method, the political optimizer [29] (PO) method by using MATLAB is used to design the PI parameters for PSS. The PO is considered one of the best recent optimizations techniques because it has a formidable execution on solving engineering optimization problems additional to complex multimodal functions. PO is inspired by human behaviours within the multi-stage political process.

PO follows a novel position updating strategy called recent past-based position updating strategy which is the mathematical modelling of the learning behaviours of the politicians from the previous election. And design the mathematical mapping of all the main steps of politics.

PO has proven [30] its superiority when compared with Whale Optimization Algorithm (WOA), Ant Lion Optimizer (ALO), Gray Wolf Optimizer (GWO), Moth Flame Optimization (MFO), MultiVerse Optimizer (MVO), Sine-Cosine Algorithm (SCA)), and Slap Swarm Algorithm (SSA). through experiments that PO has an excellent convergence speed with good exploration capability in early iterations.

By using the m-file of PO:

1- assuming the upper and the lower limiter (0.001-100) for the possible values of ( $\left.K_{p}, K_{i}\right)$.

2- Define Objective function equation the optimum damping ratio from required eigen value of $\left(\Delta \omega_{r}, \Delta \theta_{r}\right)$.

$\lambda_{\omega_{r}, \theta_{r}}=\sigma \pm j \omega$

(27) 
(28)

$$
\zeta=\sqrt{\frac{1}{1+\left(\frac{\omega}{\sigma}\right)^{2}}}
$$

Objective function equation $(0)=\frac{1}{\zeta}$

3- Define the number of iterations (100) and runs (15).

4- run $\mathrm{m}$-file to get the best value for pi parameters to get the desired the eigen-value $\left(\Delta \omega_{r}, \Delta \theta_{r}\right)$ and make all parameters of SG to be stable.

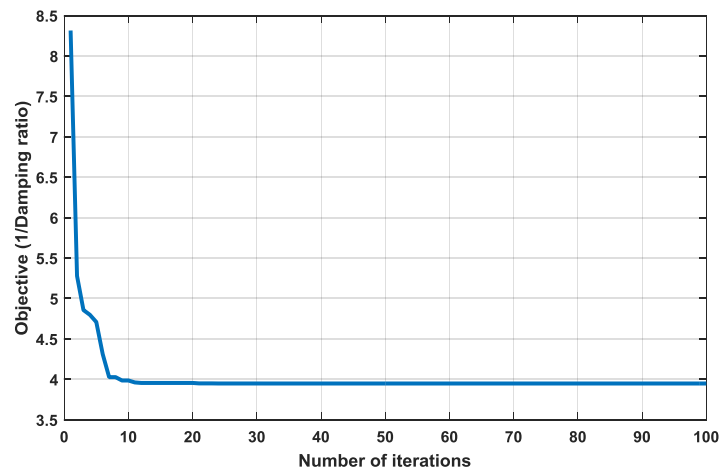

Fig 7.PO optimization for SG with damper winding

Table 1. the optimal values for PI parameters for SG with damper winding

\begin{tabular}{lcc}
\hline & $\begin{array}{c}\text { Single pole } \\
\text { placement }\end{array}$ & Political optimizer \\
\hline$K_{p}$ & 0.1095 & 0.1548 \\
$K_{i}$ & 0.0014 & 0.001 \\
$\lambda_{\omega_{r}, \theta_{r}}$ & -2.1982 & -2.9559 \\
Damping ratio $\zeta$ & $\pm j 11.199$ & $\pm j 11.29$ \\
\hline
\end{tabular}

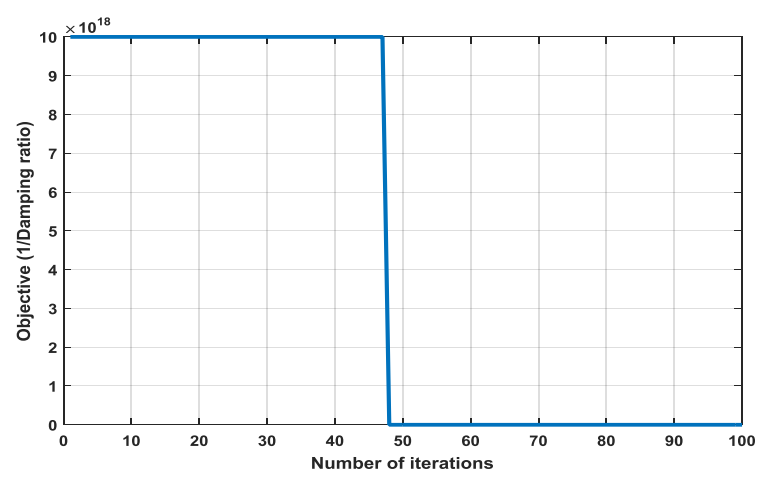

Fig 8.PO optimization for SG without damper winding
Table 2. the optimal values for PI parameters for SG without damper winding

\begin{tabular}{lcc}
\hline & $\begin{array}{c}\text { Single pole } \\
\text { placement }\end{array}$ & Political optimizer \\
\hline$K_{p}$ & 0.2459 & 0.2604 \\
$K_{i}$ & 0.0061 & 0.0011 \\
$\lambda_{\omega_{r}, \theta_{r}}$ & -2.1982 & -2.3484 \\
Damping ratio $\zeta$ & $\pm j 8.1425$ & $\pm j 8.373$ \\
& 0.2527 & 0.27
\end{tabular}

From Table1, 2.the damping ratio is improved by using PO optimizer.

after getting the best PI parameter, Linearization PSS and AVR system at small changes from steady state, rewriting the block diagrams of PSS and AVR after simplifying the system at linearization process as shown in Fig.9.

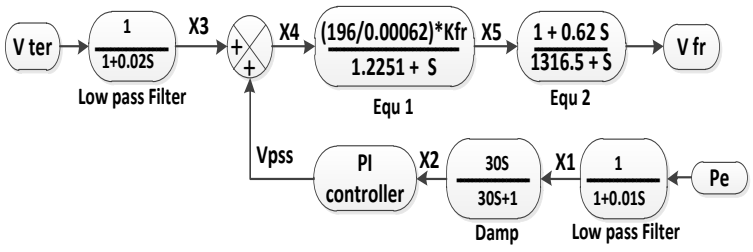

Fig 9. Block diagram after simplifying the exciter system with PSS system.

convert the equation of electromagnetic power to be per unit by dividing it to base power $p_{b}$

$\frac{p_{e}}{p_{b}}=\frac{2}{P} \quad \omega_{r} T_{e} \frac{1}{p_{b}}$

(29)

at small changes for electromagnetic power at normal operation

$\Delta p_{e}=\Delta i_{q s} a_{-} p_{t}+\Delta i_{d s} b_{-} p_{t}+\Delta i_{d r} c_{-} p_{t}+$

$\Delta i_{q r} d_{-} p_{t}+\Delta i_{f r} e_{-} p_{t}+\Delta \omega_{r} f_{-} p_{t}$

Where,

$a_{-} p_{t}=\frac{2}{P} \frac{1}{p_{b}} c_{t}\left[\psi_{d s 0}+x_{q s} i_{d s 0}\right] \omega_{r 0}$

$a_{-} p_{t}=\frac{2}{P} \frac{1}{p_{b}} c_{t}\left[\psi_{d s 0}+x_{q s} i_{d s 0}\right] \omega_{r 0}$

$b_{-} p_{t}=\frac{-2}{P} \frac{1}{p_{b}} c_{t}\left[x_{d s} i_{q s 0}+\psi_{q s 0}\right] \omega_{r 0}$

$c_{-} p_{t}=\frac{2}{P} \frac{1}{p_{b}} c_{t} x_{m d} i_{q s 0} \omega_{r 0}$

$d_{-} p_{t}=\frac{-2}{P} \frac{1}{p_{b}} c_{t} x_{m q} i_{d s 0} \omega_{r 0}$

$e_{-} p_{t}=\frac{2}{P} \frac{1}{p_{b}} c_{t} x_{m d} i_{q s 0} \omega_{r 0}$

$f_{-} p_{t}=\frac{2}{P} \frac{1}{p_{b}} T_{e 0}$

from Fig (9). first, analysis of the T.F for the block diagram of $\frac{\Delta x_{1}}{\Delta p_{e}}$ and substitute $\Delta p_{e}$ from equation (30) 
So, equation (31) will be:

$-\frac{p}{\omega_{b}}\left(0.01 \omega_{b} \Delta x_{1}\right)-\Delta x_{1}+\Delta i_{q s} a_{-} p_{t}+$

$\Delta i_{d s} b_{-} p_{t}+\Delta i_{d r} c_{-} p_{t}+\Delta i_{q r} d_{-} p_{t}+\Delta i_{f r} e_{-} p_{t}+$

$\Delta \omega_{r} f_{-} p_{t}=0$

second, analysis of the T.F for the block diagram of $\frac{\Delta v_{p s s}}{\Delta x_{1}}$, the equation (32) will be:

$\frac{p}{\omega_{b}}\left(30 k_{p} \omega_{b} \Delta x_{1}\right)+30 k_{i} \Delta x_{1}-$

$\frac{p}{\omega_{b}}\left(30 \omega_{b} \Delta v_{p s s}\right)-\Delta v_{p s s}=0$

Third, analysis of the T.F for the block diagram of

$\frac{\Delta x_{5}}{\Delta x_{4}}$, the equation (33) will be:

$\frac{p}{\omega_{b}}\left(\omega_{b} \Delta x_{5}\right)+1.2251 \Delta x_{5}-\left(\frac{196}{0.00062}\right) k_{f r} \Delta v_{p s s}-$

$\left(\frac{196}{0.00062}\right) k_{f r} \Delta x_{3}=0$

Fourth, analysis of the T.F for the block diagram of $\frac{\Delta v_{f r}}{\Delta x_{5}}$, the equation (34) will be:

$\frac{p}{\omega_{b}}\left(\omega_{b} \Delta v_{f r}\right)+1316.517 \Delta v_{f r}-$

$\frac{p}{\omega_{b}}\left(0.62 \omega_{b} \Delta x_{5}\right)-\Delta x_{5}=0$

fifth, analysis of the T.F for the block diagram of $\frac{\Delta x_{3}}{\Delta v_{t}}$ and substitute $\Delta v_{t}$ from equation (22), the equation

(35) will be:

$a_{-} v_{t} \Delta \delta_{b}+b_{-} v_{t} \Delta v_{b}=$

$-c_{-} v_{t} \Delta \theta_{r}-d_{-} v_{t} \Delta \omega_{r}-e_{-} v_{t} \Delta i_{d s}-f_{-} v_{t} \Delta i_{q s}-$

$\frac{v_{d s 0}}{v_{t 0}} \frac{x_{e}}{v_{t 0}} \frac{p}{\omega_{b}} \Delta i_{d s}-\frac{v_{q s 0}}{v_{t 0}} \frac{x_{e}}{v_{t 0}} \frac{p}{\omega_{b}} \Delta i_{q s}+$

$\frac{p}{\omega_{b}} 0.02 \omega_{b} \Delta x_{3}+\Delta x_{3}=0$

From previous equations (10-14), (17-18), (31-35) respectively are equivalent to the matrix expression to analysis the dynamic behaviour for SG with damper winding and without damper winding after removing equations $(12,13)$ and conclude the eigen values of deviation of rotor angle, rotating speed and the step response for T.F $\frac{\Delta \omega_{r}}{\Delta T_{l}}$ and $\frac{\Delta T_{e}}{\Delta T_{l}}$ at disturbance of electromagnetic load in the electric grid.

1- Get the state space of synchronous machine with exciter system (AVR and PSS)

to get state and input matrices so $\Delta v_{b}, \Delta \delta_{b}, \Delta T_{l}$ are selected as input

$\Delta \dot{x}=A * \Delta x+B * \Delta u$

$\Delta x:($ state vector $), \Delta u:($ control vector $)$
2- it is necessary to identify the desired output by forming the measurement equation:

$y=C \Delta x+D \Delta u$

$D=0$, C: output matrix

let the change in rotor speed $\Delta \omega_{r}$, the change in electromagnetic torque $\Delta T_{e}$ are selected as output

\section{Results and Effect of Disturbance Load on SG}

In this part, discuss the results of eigen values and state space for T.F $\frac{\Delta \omega_{r}}{\Delta T_{l}}$ and $\frac{\Delta T_{e}}{\Delta T_{l}}$ of SG without and with damper winding at disturbance of electromagnetic power at sudden change in load of generated power $100 \mathrm{MW}$ in the electric grid using a parameter of SG (555 MVA) [2] as shown in Table.3.

\begin{tabular}{cl} 
Table 3. synchronous generator parameter (555 MVA) \\
\hline parameter & value \\
\hline$r_{s}$ & $0.0031 \Omega$ \\
$r_{f r}$ & $0.0715 \Omega$ \\
$r_{d r}$ & $0.02947 \Omega$ \\
$r_{q r}$ & $0.006424 \Omega$ \\
$l_{l s}$ & $0.4129 * 10^{-3} \mathrm{H}$ \\
$l_{l d r}$ & $0.4716 * 10^{-3} \mathrm{H}$ \\
$l_{l q r}$ & $1.9964 * 10^{-3} \mathrm{H}$ \\
$l_{m d}$ & $4.5696 * 10^{-3} \mathrm{H}$ \\
$l_{m q}$ & $4.432 * 10^{-3} \mathrm{H}$ \\
$p_{b}$ & $499.5 * 10^{6} \mathrm{watt}$ \\
$P$ & 2 pole \\
$J$ & $27547.8 \mathrm{~N} . \mathrm{m}^{2}$ \\
$v_{l l}$ & $24000 \mathrm{volt}$ \\
$F$ & $50 \mathrm{HZ}$ \\
\hline & \\
\hline &
\end{tabular}

\subsection{Eigen values and step responses for $S G$ without damper winding}

It is necessary to get the eigen values of $\left(\Delta \omega_{r}, \Delta \theta_{r}\right)$ to study the dynamic performance of (damping ratio $\zeta$ and frequency of damping $\omega_{d}$ ) of SG at constant field voltage, AVR system and AVR \& PSS system from state matrix of previous equations of SG without equation $(12,13)$ of damper winding and by using MATLAB 
Table 4. Eigen value of rotor angle and speed of SG without damper winding

\begin{tabular}{lcl}
\hline $\begin{array}{l}\text { System of Field } \\
\text { voltage }\end{array}$ & $\begin{array}{l}\text { Eigen value of } \\
\Delta \omega_{r}, \Delta \theta_{r}\end{array}$ & Status \\
\hline Constant field voltage & $-0.0003 \pm j 8.6441$ & stable \\
& $\omega_{d}=1.3758 \mathrm{HZ}$ & \\
& $\zeta=3.4706 * 10^{-5}$ & \\
AVR system & $+0.189 \pm j 8.632$ & unstable \\
AVR \& PSS system & $-2.3484 \pm j 8.373$ & Stable \\
& $\omega_{d}=1.332 \mathrm{HZ}$ & \\
& $\zeta=0.27$ & \\
\hline
\end{tabular}

From Table.4 At SG without damper winding, the damping ratio is increased from $3.4706 * 10^{-5}$ at constant field voltage to 0.27 at PSS system and it got worse at AVR.

So, the great effect of adding PSS system to AVR because it reinforces the damping and make transient stability in the generated electromagnetic power.

As show in Fig.10, the rotor speed and electromagnetic torque of SG is stable after 20000 second but this time is too long to make the dynamic behaviours of electric grid to be critical stable

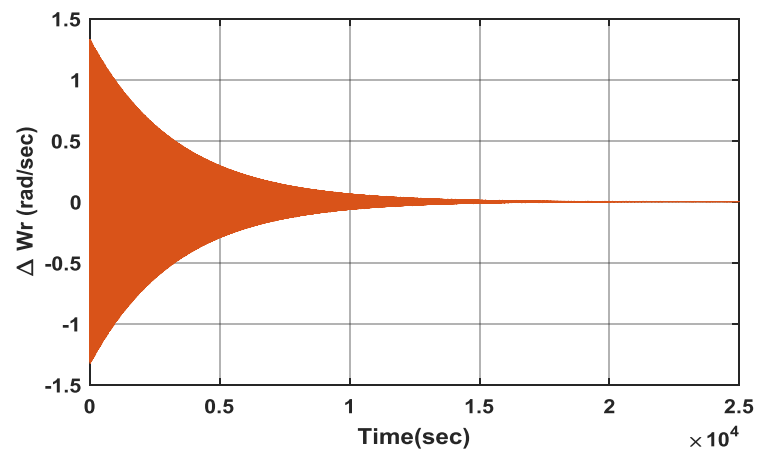

(a)

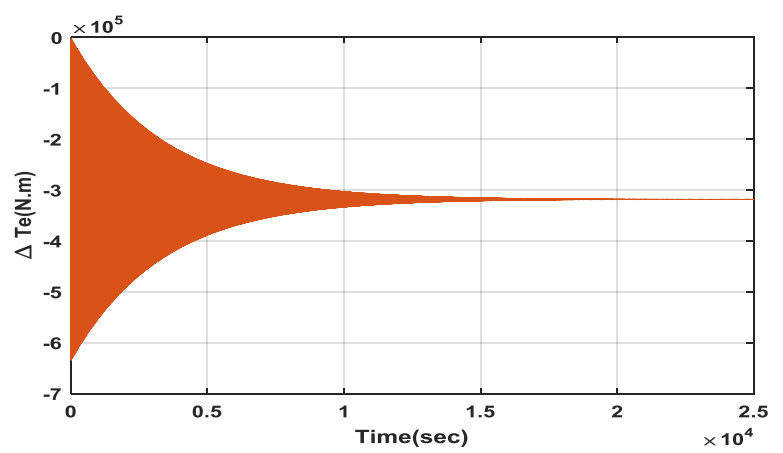

(b)

Fig 10. the analysis of rotor speed and electromagnetic torque at disturbance on the generator without damper at constant field voltage
After adding AVR system, SG became unstable as shown in fig (11)

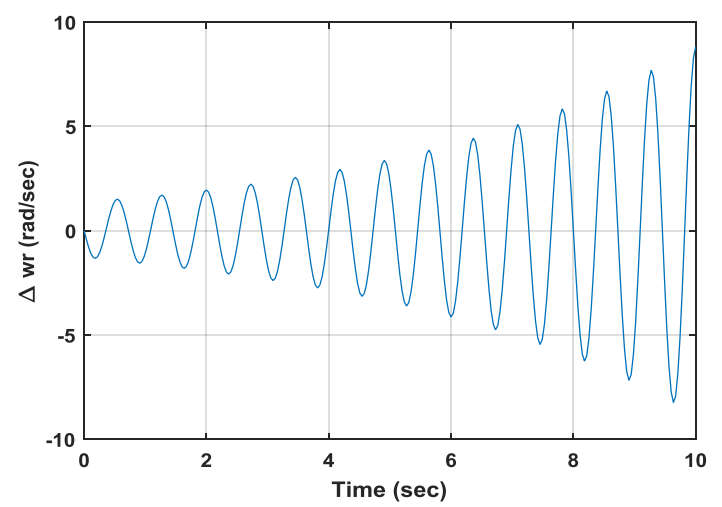

Fig 11. the analysis of rotor speed at disturbance on the generator without damper at AVR system

with PSS system and the new parameters of pi controller, SG became more stable after 2 second as shown in Fig.12.

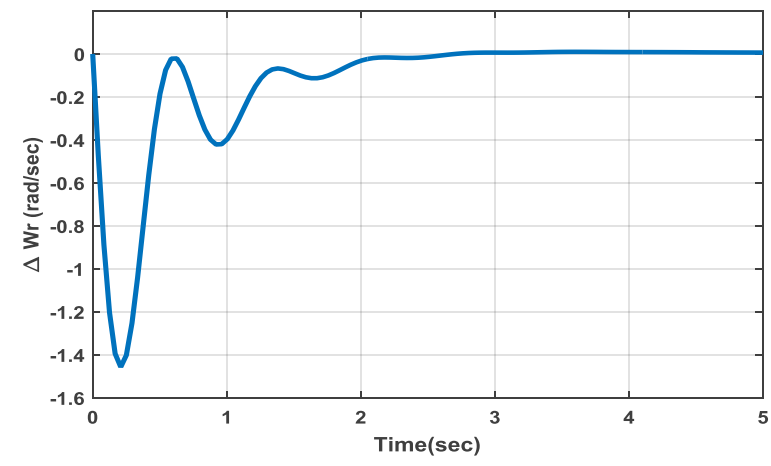

(a)

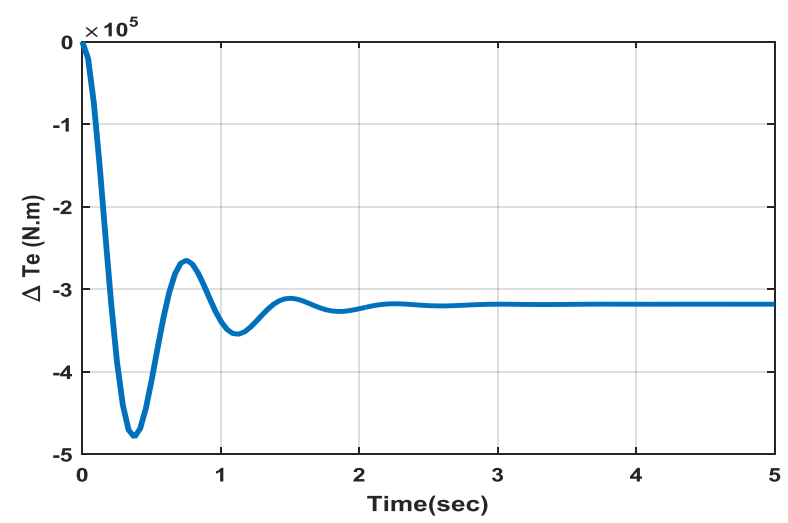

(b)

Fig 12. the analysis of rotor speed and electromagnetic torque at disturbance on the generator without damper at PSS system. 


\subsection{Eigen values and step responses for $S G$ with damper winding}

In this part, discuss the important of damper winding because it help SG to damp a small fraction of oscillations with using PSS system in SG

Table 5. Eigen value of rotor angle and speed of SG with damper winding

\begin{tabular}{lcc}
\hline $\begin{array}{l}\text { System of Field } \\
\text { voltage }\end{array}$ & $\begin{array}{l}\text { Eigen value of } \\
\Delta \omega_{r}, \Delta \theta_{r}\end{array}$ & Status \\
\hline Constant field voltage & $-1.4491 \pm j 11.092$ & stable \\
& $\omega_{d}=1.7654 \mathrm{HZ}$ & \\
& $\zeta=0.1295$ & \\
AVR system & $-1.328 \pm j 10.962$ & stable \\
& $\omega_{d}=1.7446 \mathrm{HZ}$ & \\
& $\zeta=0.1203$ & \\
AVR \& PSS system & $-2.9559 \pm j 11.29$ & Stable \\
& $\omega_{d}=1.797 \mathrm{HZ}$ & \\
$\zeta=0.2533$ &
\end{tabular}

From Table.5. At SG with damper windings, the damping ratio is decreased from 0.1295 at constant field voltage to 0.1203 at AVR system and increased to 0.2533 at PSS system

So, the damper winding and PSS system play an important role to damp the oscillations in the electrical grid. As shown in Fig .13.

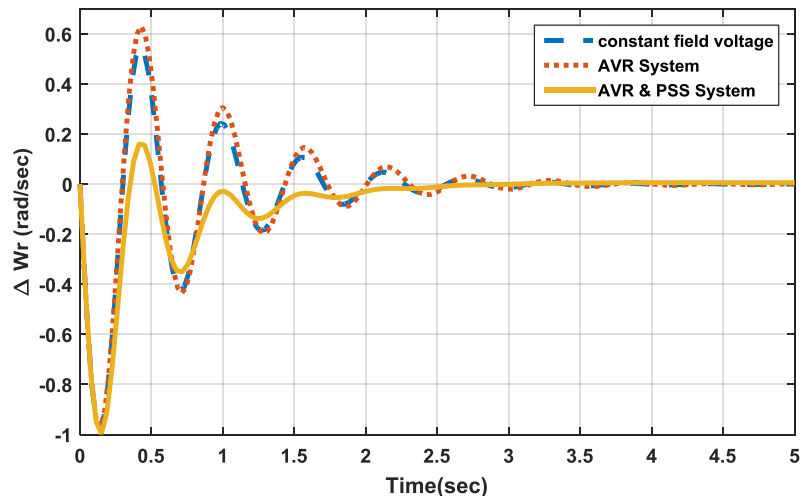

(a)

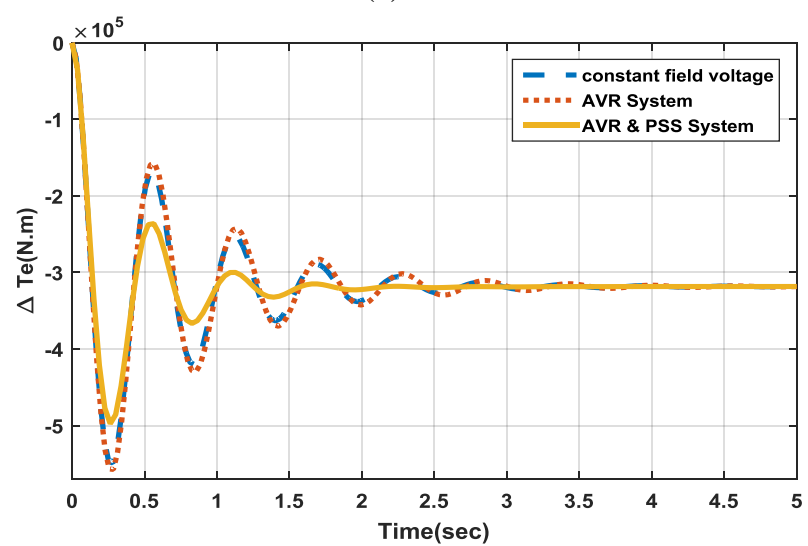

(b)

Fig 13. the analysis of rotor speed and electromagnetic torque at disturbance on the generator with damper winding at constant field voltage ,AVR and AVR\&PSS.

From Fig.13. the damping weakened by AVR and improved after using PSS and SG became more stable.

\section{Conclusions}

Based on the points described previously ,the analysis the effect of exciter system and the step response results and eigen values it is possible to conclude that:

- The damper winding in SG helps PSS system to damp the oscillations $(12.03 \%$ $25.33 \%)$ and enhance the dynamic performance.

- $\quad$ The excitation system (AVR) decreases the damping ratio and make the synchronous generator without damper winding to be unstable.

- The electromagnetic of the generated power from SG is used as the best input signal to PSS to supply affirmative influence for the damping and control the passive damping of AVR.

- From the simulation results, PSS can treatment the instability problem during oscillations for SG without damper winding and This will be economical in terms of raw materials used in the manufacture of synchronous generators.

\section{List of symbols}

\begin{tabular}{|c|c|}
\hline $\mathrm{f}$ & frequency $(50 \mathrm{HZ})$ \\
\hline$l_{l s}$ & portion of the leakage inductance which in stator \\
\hline$l_{l d r}$ & $\begin{array}{l}\text { portion of the leakage inductance which in rotor } \\
\text { of d-axis }\end{array}$ \\
\hline$l_{l q r}$ & $\begin{array}{l}\text { portion of the leakage inductance which in rotor } \\
\text { of q-axis }\end{array}$ \\
\hline$l_{l f r}$ & $\begin{array}{l}\text { portion of the leakage inductance which in field } \\
\text { of the rotor }\end{array}$ \\
\hline$l_{m d}$ & portion of the mutual inductance in d-axis \\
\hline$l_{m q}$ & portion of the mutual inductance in q-axis \\
\hline$r_{s}$ & stator resistance \\
\hline$r_{f r}$ & field resistance \\
\hline$r_{d r}$ & rotor resistance of d-axis \\
\hline$r_{q r}$ & rotor resistance of q-axis \\
\hline$P$ & number of poles \\
\hline$\omega_{r}$ & rotating speed \\
\hline $\boldsymbol{\theta}_{r}$ & rotor angle \\
\hline
\end{tabular}




\begin{tabular}{|c|c|}
\hline$\omega_{b}$ & base electromagnetic angular velocity \\
\hline$p$ & differentiation for time $\frac{d}{d t}$ \\
\hline$T_{e}$ & electromagnetic torque \\
\hline$T_{l}$ & mechanical torque \\
\hline$J$ & moment of inertia \\
\hline$r_{e}$ & resistance of line \\
\hline$l_{e}$ & inductance of line \\
\hline$v_{b}$ & infinite bus voltage amplitude \\
\hline$\theta_{b}$ & Infinite bus phase \\
\hline$i_{d s}$ & d-axis stator current \\
\hline$i_{q s}$ & q-axis stator current \\
\hline$i_{d r}$ & d-axis rotor current of damper winding \\
\hline$i_{q r}$ & $\mathrm{q}$-axis rotor current of damper winding \\
\hline$i_{f r}$ & current of field winding \\
\hline$v_{d s}$ & d-axis stator voltage \\
\hline$v_{q s}$ & q-axis stator voltage \\
\hline$v_{d r}$ & d-axis rotor voltage of damper winding \\
\hline$v_{q r}$ & $\mathrm{q}$-axis rotor voltage of damper winding \\
\hline$v_{f r}$ & voltage of field winding \\
\hline$v_{t}$ & terminal voltage (peak - phase) \\
\hline$I_{t}$ & terminal current (peak - phase) \\
\hline$\phi$ & Power factor angle \\
\hline$\delta$ & Load angle \\
\hline$p_{b}$ & base power \\
\hline $\boldsymbol{p}_{e}$ & electromagnetic power \\
\hline PSS & Power system stabilizer \\
\hline AVR & automatic voltage regulation \\
\hline $\boldsymbol{k}_{p}$ & Proportional coefficient, PI controller \\
\hline $\boldsymbol{k}_{\boldsymbol{i}}$ & Integral coefficient, PI controller \\
\hline$\omega_{d}$ & Frequency of damping \\
\hline$\lambda$ & Eigen Value \\
\hline
\end{tabular}

signal stability", Electric Power Systems Research Vol. 181, April 2020

[8] S.S.Venkata, E.Mircea, and T.Lucian, "BACKGROUND OF POWER SYSTEM STABILITY", Handbook of Electrical Power System Dynamics: Modeling, Stability, and Control, pp. 453475, 2013.

[9] H.E.Mostafa, M.A.El-Sharkawy, A.A.Emary, K.Yassin, "Design and allocation of power system stabilizers using the particle swarm optimization technique for an interconnected power system", Electric Power Energy System Vol. 34, pp. 57-65, 2012.

[10] M.Tofighi, M.Alizadeh, S.Ganjefar, M.Alizadeh, "Direct adaptive power system stabilizer design using fuzzy wavelet neural network with self-recurrent consequent part", Applied Soft Computer Vol. 28, pp. 514-526, 2015.

[11] J.Talaq, "Optimal power system stabilizers for multi machine systems", International Journal of Electrical Power \& Energy Systems Vol. 43, pp. 793-803, 2012.

[12] A.Khodabakhshian, R.Hemmati and M.Moazzami, "Multi-band power system stabilizer design by using CPCE algorithm for multi-machine power system ", Electric Power System Research Vol. 101, pp. 36-48, 2013.

[13] L.H.Hassan, M.Moghavvemi, H.F.Almurib, K.M Muttaqi, V.G.Ganapathy, "Optimization of power system stabilizers using participation factor and genetic algorithm", Electric Power Energy System Vol. 55, pp. 668-679, 2014.

[14] N.Fischer, G.Benmouyal, " Tutorial on the Impact of the Synchronous Generator Model on Protection Studies", Journal of Reliable Power Vol. 3, pp. 1-18, 2012.

[15] M.DEHGHANI and M.KARRARI, "nonlinear robust modeling of synchronous generators ", Iranian Journal of Science \& Technology Vol. 31, pp. 629-640, 2007.

[16] H.Bourles and T.Margotin, "Analysis and design of robust coordinated AVR/PSS", IEEE transaction on power systems Vol. 13, pp. 568-575, 1998.

[17] W.B.Chu, J.M.Liu, J.Z.Liu, and L.X.Tan, "Modeling and Simulation about Included New PSS of Excitation System", Electric Machines and Control Application, 2009.

[18] X.F.Wang, W.L.Fang, and Z.C.Du, "Modern Power System Analysis", Science Press, 2003.

\section{References}

[1] A.A.zea, "Power System Stabilizers for The Synchronous Generator", CHALMERS UNIVERSITY OF TECHNOLOGY, 2013.

[2] P.M. Anderson and A.A. Fouad, "Power System Control and Stability", John Wiley (IEEE Press), 2003.

[3] L. Angquist and C. Gama, "Damping algorithm based on phasor estimation", Power Engineering Society Winter Meeting (IEEE), 2001.

[4] L.M.Hajagos and M.J.Basler, "Changes to IEEE 421.5 recommended practice for excitation system models for power system stability studies", IEEE Power Engineering Society General Meeting, 2005.

[5] T.A.Lipo, "Analysis of synchronous machines", CRC press, 2017.

[6] Kundur and Taylor, "Power system stability and control", McGraw-Hill, 1994.

[7] G.Samundra , J.Francisco and N.Sumate , " Comparative analysis of probabilistic and deterministic approach to tune the power system stabilizers using the directional bat algorithm to improve system small-

[19] H.L.Yang, T.Y.Zhang, and J.J.Guo, "Action of PSS on system stability and parameter establishment in field regulator", North China Electric Power, 2005.

[20] M.Huang, and H.Y.Han, "The PSS parameters optimization based-on power system operating variables", Electric Power, 2007.

[21] F.Zhang, J.F.Li, W.Wang, H.P.Zhou, Z.H.Xiang, G.S.Ye, and Y.P.Zhou, "The simulation of PSS and SVC to stability of electric power transmission based on MATLAB", Electric Switchgear, 2009.

[22] P.Kundur and Others, "A pss tuning toolbox and its applications" Power Engineering Society, General Meeting, 2003.

[23] D.MELLO," Concepts of Synchronous Machine Stability as Affected by Excitation Control", IEEE Transactions on Power Apparatus and Systems, 1969.

[24] X.Chen, J.Hu, K.Chen and Z.Peng, " Modeling of electromagnetic torque considering saturation and magnetic field harmonics in permanent magnet synchronous motor for HEV", Simulation Modelling Practice and Theory Vol. 66, pp. 212-225, 2016. 
[25] M.Babaei, R.Asgharei and A.Ahmarinejad, "Electromagnetic Torque and Speed Estimators for Permanent Magnet Synchronous Motor Drive Systems", Energy Procedia Vol. 100, pp. 291-296, 2016.

[26] J.Talaq, "Optimal power system stabilizers for multi machine systems", International Journal of Electrical Power \& Energy Systems Vol. 43, pp. 793-803, 2012.

[27] P.K.Ray and S.R.Paital, "A robust power system stabilizer for enhancement of stability in power system using adaptive fuzzy sliding mode control", Applied Soft Computing Vol. 73, pp. 471-481, 2018.

[28] S.Gomes, C.H.C.Guimarães and N.Martins, "Damped Nyquist Plot for a pole placement design of power system stabilizers", Electric Power Systems Research Vol. 158, pp. 158-169, 2018.

[29] Q.Askari, I.Younas and M.Saeed, "Political Optimizer: A novel socio-inspired meta-heuristic for global optimization", Knowledge-Based Systems Vol. 195, pp. 205-240, 2020.

[30] Q.Askari and I.Younas, "Political Optimizer Based Feedforward Neural Network for Classification and Function Approximation", Neural Processing Letters Vol. 1, pp. 80-111, 2021. 